\title{
Paridad de género en las organizaciones internacionales: discurso vs. números
}

\section{Enzo Lenine y Manuela Pereira*}

\begin{abstract}
RESUMEN
Aunque el debate sobre la presencia femenina en los espacios políticos haya empezado antes de los años setenta del siglo veinte, el contexto de las Conferencias Mundiales sobre la Mujer profundizó las demandas de las mujeres en distintos dominios, entre ellos la participación en las esferas políticas y de decisión. Las Naciones Unidas han fomentado discursos sobre el empoderamiento de las mujeres y su participación activa en roles políticos, reconociendo que la paridad de género en la política es un objetivo esencial para alcanzar la igualdad entre hombres y mujeres. Sin embargo, la propia organización no cumple su discurso: hasta el presente momento, no hay registros de mujeres que hayan ocupado el cargo de Secretaria General y pocas ocupan otros cargos en la organización. Un escenario semejante se observa en las demás organizaciones internacionales, especialmente las que se ocupan de temas económicos, considerados de alto prestigio en la arena internacional.
\end{abstract}

En este artículo, nuestro objetivo consiste en investigar la presencia femenina en los órganos de Naciones Unidas y organizaciones económicas internacionales. Tal investigación se centra en dos enfoques: por un lado, contabilizamos la presencia femenina en distintos organismos a lo largo de tres décadas, siguiendo un abordaje tradicional de los estudios de género: counting women to make women count; por otro lado, utilizamos los datos recolectados para evaluar cómo las estructuras de género en los organismos internacionales operan en detrimento de las mujeres. Para esto, iniciamos con la siguiente cuestión: ¿Cuál es el actual estado de participación de las mujeres en los más altos cargos de la Organización de las Naciones Unidas y de los organismos del sistema económico global?

Para responder a esta pregunta, estructuramos el artículo en cuatro secciones. Primeramente, presentamos el contexto de la presencia femenina en las organizaciones internacionales y de las Conferencias Mundiales sobre la Mujer. Enseguida discutimos los ejes teóricos que informan nuestras interpretaciones. Adoptamos una perspectiva teórica feminista que dialoga con los principales ejes conceptuales manifestados en las disciplinas de las Relaciones Internacionales y la Ciencia Política. En la tercera sección, discutimos nuestro diseño metodológico de investigación. En la cuarta sección, presentamos datos estadísticos del sistema de Naciones Unidas y de instituciones económicas internacionales entre 1990-2018 acerca de la participación de las mujeres en los cargos de presidencia y vicepresidencia de órganos y organismos especializados, argumentando que la presencia de mujeres en dichos espacios sigue baja. Las teorías feministas nos permiten evaluar la presencia más allá de los datos cuantitativos, ofreciendo una lectura acerca de los roles esperados de las (pocas) mujeres que ocupan espacios de liderazgo en las organizaciones internacionales. Verificamos que las mujeres suelen ocupar cargos asociados a temas de la baja política y el cuidado, como los asuntos sociales, de infancia y alimentos, lo que refuerza roles y jerarquías de género. Además, los datos demuestran que las pocas mujeres que ocupan estos cargos provienen principalmente de América del Norte y Europa Occidental, lo que limita las perspectivas feministas que acceden a las agendas internacionales.

\section{Palabras clave}

Mujeres; género; Naciones Unidas; organizaciones económicas internacionales; Relaciones Internacionales; paridad de género; política internacional.

\section{TitLE}

Gender parity in international organizations: discourses v numbers

\section{Extended Abstract}

Throughout the history of contemporary feminism, women's presence in the public sphere has been a perennial issue,
* Enzo LENINE, Doctor en Ciencia Política por la UFRGS (Brasil). Profesor en el grado de Relaciones Internacionales del Instituto de Humanidades y Letras de la Universidad de la Integración Internacional de la Lusofonía AfroBrasileña (UnilabMalês, Brasil). Contacto: lenine@ unilab.edu.br

Manuela PEREIRA

Licenciada en Relaciones Internacionales en la Universidad de la Integración Internacional de la Lusofonía AfroBrasileña (UnilabMalês, Brasil) y estudiante en la Maestría de Estudios de Desarrollo en el Instituto Universitário de Lisboa (Portugal). Contacto: 7336manu@gmail. com

Recibido:

23.06.2020

Aceptado:

09.07.2021

DOI:

https://doi.org//0.15366/relacionesinternacionales202I.48.006

Formato de citación recomendado:

LENINE, Enzo y PEREIRA, Manuela (202I). "Paridad de género en las organizaciones internacionales: discurso vs. números", Relaciones Internacionales, n 48 , pp. I01-121. 
demanding all sorts of strategies to promote women's inclusion in the economic and political arenas. Starting in the 1980s, feminist scholars in International Relations have questioned the absence of women in the international arena, alongside the pervasive gender hierarchies of the international system. Not only was it that women had been systematically excluded from politics, but the scholarship in IR had largely ignored feminist claims about the gendering of international politics. Largely animated by feminist movements and the nascent feminist debates in IR, the World Conferences on Women (1975, 1980 , 1985, and 1995) stressed the paramount need for taking women's demands seriously. Such demands encompassed social, economic, and political domains, echoing decades of feminist struggles in the first, second and third worlds. The United Nations played a crucial role in fostering an agenda of gender equality and women's rights as human rights, which have been a quintessential part of the Millennium Development Goals and, more recently, the Sustainable Development Goals.

Furthermore, gender-oriented policies have been promoted in myriad UN agencies and international organisations, such as the World Bank and International Monetary Fund. Despite all these efforts purporting to raise awareness about gender equality and women's rights, as well as about the need for more inclusive policies for women in the international arena, women's presence in international fora as leaders has been remarkably low.As for the United Nations, until now no woman has been elected to the organisation's highest post, that of secretary general, and only recently the International Monetary Fund and World Bank have had female managing directors. These sobering results show a different picture of international organisations that promote gender equality as their political and social commitment, whilst failing to comply with the very same discourse when it comes to women's leadership in their formal structures. In this context, the present article aims to assess quantitatively and qualitatively women's presence in leadership roles at the United Nations and international economic organisations (namely, the IMF,WB and World Trade Organisation). It departs from the following research question:What is the current state of affairs of women's participation in the highest posts of the United Nations and the international organizations of the global economic system? Methodologically, we resort to descriptive statistical data of women's presence at various agencies of the United Nations and the aforementioned economic institutions from 1990-2018. Of greatest concern will be those occupying the posts of presidency and vice-presidency.

Counting women is a traditional approach in feminist studies in IR, and it is a paramount step in making women count. The second goal of our paper, thus, derives directly from the data: by mapping the few women occupying positions of leadership in international organisations, we can assess the gender structures operating to the disadvantage of women. In order to do so, we resort to feminist theories as developed in political science and IR, for they provide the conceptual tools to provide data with meaning. We focus primarily on how gendered institutions confine women to specific gendered roles that emanate from the private sphere of domesticity. This means that women suffer from various exclusionary dynamics: firstly, they are excluded from leadership roles for the fact of being women; secondly, the few who manage to break through the glass ceiling of a masculine international arena are assigned positions that mimic the elements of the private sphere. Frequently, female leaders are responsible for social issues, childhood, food security, education, and culture, all of which are labeled as soft issues, or belonging to the domain of low politics.

Feminist IR scholars have extensively denounced these gendered structures as part of how men preserve their privileges under the framework of hegemonic masculinity. Therefore, we draw on the work of several IR feminists who are concerned with the theoretical questioning and empirical unravelling of such gender hierarchies to interpret our data beyond the mere counting of women. We contend that women in leadership roles in the United Nations and international economic organisations more frequently occupy portfolios that are seen as soft issues or low politics. Likewise, these roles are often associated with the care for others, which reinforces gender roles and hierarchies. Women are more likely to occupy the presidencies and vice-presidencies of agencies such as UNICEF, UNESCO,WFP, and OHCHR, all of which deal with issues associated with childhood, education, culture, food security and human rights. We conclude that an apparent glass ceiling the invisible barrier that prevents women from reaching higher and more prestigious posts in their professional careers - still remains in international institutions, limiting the participation of women in decision-making fora.The few female leaders who manage to reach the highest posts in the international arena are more likely to be exceptions of a pervasive phenomenon of gender inequality and lack of representativeness. The discourses embraced by the United Nations and international economic organisations fail to meet the minimal criteria for gender parity within these organizations' structures, and even where women have been assigned leadership roles, gender stereotypes still prevail. Further evidence revealed in our research refers to the regional representativeness of the few female leaders in these international organisations: about $55 \%$ of them come from Western Europe and North America. Latin American and African women are strongly underrepresented in the United Nations system, as well as in the Bretton Woods institutions, which is itself problematic because the particular perspectives of women from the Global South are also excluded from debates in these international fora.

Therefore, international organisations also face the challenge of fostering the diversity of feminist perspectives by developing strategies to include Global South women in their formal structure, ideally as leaders. In this sense, our paper draws attention to the importance of pluralism not only in terms of gender parity, but also of a feminist worldview. In order to make women count in the international arena, the United Nations and international economic organisations have to fully commit themselves to concrete policies for women's inclusion, not only as heads of the institutions, but also in lower hierarchies where policies are designed. Only by acknowledging that women's perspectives matter, can we achieve the goals of gender equality.

\section{KEYWORDS}

Women; Gender; United Nations; international economic organizations; International Relations; gender parity; international politics. 


\section{ntroducción}

En su mensaje por ocasión del ocho de marzo de 2020, el Secretario General de las Naciones Unidas, António Guterres, reconoció los retos enfrentados por las mujeres en las últimas décadas, tanto en el ámbito nacional de las políticas domésticas de cada miembro de la organización como en la arena internacional, especialmente en la esfera política. Guterres, a determinada altura de su discurso, reafirma sus compromisos para con las mujeres en el contexto de la ONU:

“[...] la paridad de género en las Naciones Unidas es una de mis principales prioridades, que ya ha llevado al logro de la paridad en las categorías superiores del personal, dos años antes de lo que habíamos previsto. En el futuro, haré todo lo que esté a mi alcance para asegurarme de que las mujeres estén representadas en todos los ámbitos de toma de decisiones en las Naciones Unidas, incluidos los procesos de paz" (Guterres, 2020).

Las motivaciones detrás de tal compromiso se comprenden cuando se analizan los emblemáticos datos de ONU Mujeres. En I de enero de 2020, solamente el 6,6\% de los jefes de estado y el $6,2 \%$ de los jefes de gobierno eran mujeres; un $20,5 \%$ de las presidencias de parlamentos del mundo y un $25,3 \%$ de las vicepresidencias son ocupadas por representantes femeninas (ONU Mujeres, 2020). La presencia femenina como miembros de los parlamentos sigue tendencias semejantes: 43,9\% en los países nórdicos, 31,3\% en las Américas, 28,8\% en Europa, $24,4 \%$ en África Subsahariana, $20 \%$ en Asia, 16,6\% en Oriente Medio y África del Norte, y 19,4\% en los países del Pacíico (ONU Mujeres, 2020). Como se nota, la paridad de género en la política persiste como un reto en la mayoría de los países del mundo, incluso en aquellos donde las políticas para la promoción de la igualdad entre hombres y mujeres se encuentran históricamente más avanzadas (por ejemplo, los países nórdicos).

Sin embargo, estos datos se refieren tan solo a contextos nacionales, omitiendo datos sobre la presencia femenina en las Naciones Unidas y en otras organizaciones internacionales. Aunque el Secretario General reconozca que la representación de las mujeres en las esferas de decisión de la organización es precaria, él no deja explícito el déficit de dicha representación. ¿Cuántas mujeres hay en la ONU? ¿Cuáles son los cargos que ocupan? ¿De qué temas son responsables si llegan a una posición de decisión? ¿La presencia femenina, cuando existe, es democráticamente representativa de la diversidad de miembros de las Naciones Unidas o su origen nacional demuestra una prevalencia de determinadas regiones? Estas son cuestiones urgentes en el contexto de una organización que impulsa políticas para las mujeres por lo menos desde los años setenta del siglo veinte. Responderlas significa comprender si y cómo las mujeres ocupan la arena internacional, especialmente en el seno de la organización internacional más importante.

Bajo este contexto, este artículo ofrece una respuesta a una parte de estas preguntas, revelando los números que el Secretario General no alude en su mensaje del ocho de marzo. Nuestra inquietud consiste en la siguiente cuestión: ¿cuál es el actual estado de participación de las mujeres en los cargos más altos de la ONU y de los organismos del sistema económico 
global? Buscamos comprender cómo a lo largo de tres décadas, durante las cuales se han logrado sustantivos avances en las discusiones acerca de la igualdad de género y los derechos de las mujeres, las Naciones Unidas han ejecutado sus propios discursos de empoderamiento político de las mujeres en sus estructuras de decisión. Si la representación femenina es un objetivo común de los países y de la organización, y si el empoderamiento presume (o debería presumir) de romper el techo de cristal' que condiciona a las mujeres determinados roles de género, es fundamental que la ONU produzca avances internos, especialmente en los cargos de mayor visibilidad. Lo mismo se espera de las instituciones del sistema económico global, algunas de las cuales han sido conducidas por mujeres en el pasado reciente.

Este artículo se divide en cuatro secciones. En la primera sección, presentamos brevemente la evolución histórica de la agenda sobre las mujeres en las Naciones Unidas, enfocándonos en el contexto de las Conferencias Mundiales sobre la Mujer. Enseguida, discutimos la cuestión de las perspectivas teóricas feministas que tratan de la representación y presencia femenina en la arena internacional. En la tercera sección, presentamos la metodología de investigación, donde proponemos dos hipótesis de análisis. En la cuarta sección, discutimos los resultados a la luz de las teorías feministas. Finalmente, en la conclusión, evaluamos nuestros hallazgos ante los retos que enfrentan la ONU y demás instituciones internacionales en la promoción de una agenda de representación política femenina.

\section{Las mujeres y las Naciones Unidas: rasgos históricos y discursivos}

La historia de la presencia femenina en las Naciones Unidas remite, si no al momento inmediato de su fundación después de la Segunda Guerra Mundial, seguramente a la generación de una agenda internacional de derechos humanos (Adami, 2019, pp. 2-4, pp. 19-2I). La imagen en la cual Eleanor Roosevelt sostiene la Declaración Universal de Derechos Humanos es rápidamente reconocida en la mayoría de los libros que tratan del tema, así como su rol como primera presidenta de la Comisión de Derechos Humanos de la ONU y o su autoría de la "Carta abierta a las mujeres del mundo" (la que fue redactada con otras 17 mujeres representantes en la organización). En este documento, las pioneras en la organización proclamaron cuatro oportunidades y responsabilidades que las mujeres del mundo, junto a las Naciones Unidades, deberían confrontar en el futuro:

"Primero, reconocer el progreso que las mujeres han logrado durante la guerra y participar activamente en el esfuerzo para mejorar el nivel de vida en sus propios países y el apremiante trabajo de reconstrucción, para que haya mujeres calificadas y listas para aceptar la responsabilidad cuando surgen nuevas oportunidades;

Segundo, capacitar a sus hijos, niños y niñas por igual, para comprender los problemas mundiales y la necesidad de cooperación internacional, así como los problemas de sus

En la literatura de género, el término techo de cristal expresa la limitación velada al ascenso laboral de las mujeres dentro de las instituciones, organizaciones y empresas. En el contexto de que tratamos en este artículo, el techo de cristal en los organismos internacionales limita las posibilidades de las mujeres de ocupar roles de mayor prestigio y visibilidad pública. 
propios países;

Tercero, para no dejarse engañar por movimientos antidemocráticos ahora o en el futuro;

Cuarto, reconocer que el objetivo de una participación plena en la vida y las responsabilidades de sus países y de la comunidad mundial es un objetivo común hacia el cual las mujeres del mundo deben ayudarse mutuamente" (Naciones Unidas, 2020).

Estos nortes de acción se mantuvieron en la agenda de Naciones Unidas, aunque las distintas maneras como los hombres se impusieron ante las demandas femeninas hayan comprometido un enfoque claro y temáticamente orientado para las mujeres (Adami, 2019, pp. 4-5). El tratamiento difuso de cuestiones establecidas en el ámbito de la carta abierta no se demostró suficiente para atacar una diversidad de desigualdades y violencias que las mujeres en todas las partes del mundo sufrían de manera rutinaria. Es verdad que desde 1947 las Naciones Unidas, a través de la Comisión de la Condición Jurídica y Social de la Mujer (Commission on the Status of Women, CSW, en inglés), discute un amplio espectro de cuestiones que afligen a las mujeres de todo el mundo. Fue en el ámbito de CSW que se propuso la organización de conferencias dedicadas a profundizar las demandas feministas que ya se presentaban a través de los movimientos sociales en distintos países de Occidente y del bloque comunista (Ghodsee, 20l0, p. 4). Los años setenta fueron cruciales para que se cementara la demanda por un evento internacional para congregar a todas las mujeres del globo, lo que resultó en el establecimiento del Decenio de las Naciones Unidas para la Mujer (Bonfiglioli, 2016, p. 524; Bunch, 20I2, p. 213 ; Ghodsee, 2010, pp. 4-5).

El Decenio consistió de un arco de tres Conferencias Mundiales sobre la Mujer, realizadas en 1975, en la Ciudad de México; en 1980, en Copenhague, y en 1985, en Nairobi.Aunque el plan inicial se haya completado en este período, en 1995 se organizó una cuarta conferencia en Beijing. Los cuatro encuentros ofrecieron un espacio de debates entre distintas perspectivas acerca de las necesidades y retos de las mujeres en un mundo dividido por la Guerra Fría, y las diferencias socioeconómicas entre los países del Tercer Mundo y los demás (Bonfiglioli, 2016, Pp. 524-529; Bunch, 2012, p. 215, p. 218; Ghodsee, 2010, pp. 5-9).

En los años setenta, el cisma ideológico entre capitalismo y socialismo produjo una división entre los movimientos feministas que ocurrían en Estados Unidos y Europa Occidental, de un lado, y en la Unión Soviética y sus aliados, del otro. El feminismo liberal triunfó en el contexto estadounidense y europeo, mientras que en los países comunistas el feminismo se ocupó de las desigualdades socioeconómicas que resultan de las jerarquías sexuales (Bonfiglioli, 2016, p. 525; Ghodsee, 2010, p. 4). Sin embargo, la organización de un palco amplio y plural de discusiones brindó a una audiencia femenina de centenas de países la oportunidad única de llevar su voz y sus demandas para la arena internacional. En la conferencia de la Ciudad de México participaron I 25 delegaciones; en la de Copenhague, I 45 países enviaron sus representantes; en la conferencia de Nairobi, 157 estados estuvieron presentes; y en Beijing, 189. Además de las representaciones oficiales, las conferencias contaron con la presencia de organizaciones no gubernamentales y los medios de comunicación, actores fundamentales en un contexto de democratización de los espacios internacionales de discusión. 
Los temas del Decenio para la Mujer trataron de una multiplicidad de cuestiones relacionadas con la igualdad, el desarrollo y la paz.Aunque en cada conferencia se hayan generado distintos planes de acción y declaraciones, subyacen a ellas los rudimentos de una justicia de género centrada en tres ejes: corrección de desigualdades socioeconómicas, confrontación de las diversas formas de discriminación y violencia contra las mujeres, y representación y participación política de las mujeres (Fraser, 2013, pp. 193-200; Lenine y Oncampo, 2020, p. 3). Se reconoce, por lo tanto, que las diferentes perspectivas acerca de las demandas femeninas compartidas entre mujeres de los llamados primer, segundo y tercer mundo no impidieron que se produjeran comprensiones mínimamente consensuadas sobre las injusticias vividas por ellas en sus países.

Además de las Conferencias Mundiales dedicadas exclusivamente al tema de las mujeres, se destaca en la trayectoria de los movimientos feministas internacionales ante las Naciones Unidas el reconocimiento de los derechos de las mujeres como parte esencial de los derechos humanos (Bunch, 2012, pp. 216-219). La Conferencia Mundial de Derechos Humanos, realizada en 1993 en Viena, fue un rasgo histórico en el contexto de los esfuerzos de promoción de derechos humanos por la ONU, amplificando debates acerca de las intrincadas relaciones entre ellos, desarrollo y democracia. En su declaración y plan de acción, se cristalizó la comprensión de que los derechos de las mujeres y de las niñas necesitan mecanismos específicos de promoción y defensa en el ámbito de los derechos humanos (ACNUDH, 2020, pp. I-2).

Es en parte como resultado de los debates promovidos a lo largo de estas tres décadas que las Naciones Unidas lanzan, a comienzos del siglo XXI, una amplia agenda de desarrollo en la cual la igualdad de género y la autonomía de la mujer figuran como metas fundamentales. Los Objetivos de Desarrollo del Milenio (2000-20I5) y, posteriormente, los Objetivos de Desarrollo Sostenible (2015-2030) amplificaron las demandas de las mujeres, consolidándolas definitivamente en el tablero internacional. Como subraya el informe de ONU Mujeres:

\begin{abstract}
"Veinte años después del hito que supuso la Cuarta Conferencia Mundial sobre la Mujer, celebrada en Beijing, $y$ en un momento en el que la comunidad mundial se encuentra inmersa en la definición de los Objetivos de Desarrollo Sostenible (ODS) para la era post-2015, parece que el consenso internacional sobre la necesidad de lograr la igualdad de género es más firme que nunca. El empoderamiento de las mujeres y las niñas es uno de los objetivos más ansiados por todos, desde las organizaciones de base, los sindicatos y las empresas, hasta los estados miembros y los órganos intergubernamentales" (ONU Mujeres, 2016, p. 589).
\end{abstract}

Sin embargo, la intensificación de las demandas feministas y su consecuente ascenso en la agenda internacional no significó una inmediata transformación de la realidad de exclusión de las mujeres en prácticamente todas las esferas de la vida social, política y económica. El mismo informe de ONU Mujeres (2016) alerta de las promesas no cumplidas y metas no alcanzadas, especialmente con respecto a los derechos de las mujeres, $y$ en especial su protección a través de instrumentos y mecanismos específicos y su realización plena. Evidentemente, las Conferencias 
Mundiales sobre la Mujer y la Declaración de Viena desempeñaron un papel fundamental en la construcción de agendas dedicadas a las necesidades de las mujeres, pero el camino hacia una justicia de género aún sigue en el horizonte de las políticas domésticas e internacionales.

En este contexto, persiste un déficit de participación política de las mujeres en los distintos espacios públicos ${ }^{2}$. Como se ha relatado en la introducción, la presencia femenina en los altos cargos políticos no se acerca a la paridad de género en la mayoría de los países del mundo.Además, y según los mismos datos de ONU Mujeres (2020), las pocas mujeres que ocupan ministerios en sus países desempeñan funciones identificadas con las jerarquías de género: asuntos sociales, familia, infancia, juventud, tercera edad, medio ambiente, recursos naturales, entre otros que se reconocen como trabajos del cuidado. Se percibe, por lo tanto, que camina pari passu al déficit de representación un refuerzo de los roles de género, que atribuyen a las mujeres determinadas funciones que reflejan el espacio privado y doméstico. Detallamos esta discusión en la próxima sección.

\section{Perspectivas feministas: roles de género y representación política}

La historia del feminismo en Occidente está directamente relacionada con la efervescencia filosófica, política y social de la llustración. MaryWollstonecraft (I792[20 I8]) y Olympe de Gouges (I79I[20I7]) figuran como las primeras feministas que han registrado sus comentarios acerca de los derechos de la mujer en sus respectivas sociedades, planteando en sus reivindicaciones el reconocimiento de ellos como parte intrínseca de los movimientos por liberación de su época. Las revoluciones que caracterizan los siglos XVIII y XIX logran éxito en romper las estructuras de dominación que privaban a los hombres de su libertad, pero la expansión de los derechos se refrena ante el criterio sexual, manteniendo las jerarquías patriarcales vigentes.

Estas jerarquías estructuran prácticamente todas las sociedades desde tiempos remotos, definiendo roles y espacios específicos basados en el sexo (Biroli, 2018, p. 21; Okin, 2008, pp. 305-308; Pateman, I 988 [1993, pp. 28-29]); Segato, 2016, pp. 9l-96). Al reservar espacios sociales específicos para cada sexo, se establece un orden social en el cual hombres y mujeres tienen papeles determinados para desempeñar.Tal distinción se establece a partir de un marco concreto: el de la casa o, más generalmente, del doméstico. La metáfora es simple, pero suficientemente intuitiva: de puertas para adentro, prevalece el dominio de lo privado y de la domesticidad, donde se concreta la esfera de la mujer; al otro lado, de puertas para afuera, está la esfera pública y de lo universal, por donde transitan los hombres.

Subyacentes a esta división de espacios — que contempla una esfera pública destinada al mundo y otra privada, confinada en sí misma y su domesticidad- están las relaciones de poder que organizan el orden social (Miguel, 2019, p. 32-37). Evidentemente, tal división de espacios, que emerge del propio liberalismo, resguarda, en un momento de profundas intervenciones del poder estatal, una esfera individual que no debería estar bajo los escrutinios de la sociedad y, más precisamente, del estado. Sin embargo, cuando se reconoce que esta misma división engendra

Además de los datos de ONU Mujeres (2020) sobre mujeres en la política, es importante destacar la baja presencia femenina en uno de los espacios públicos más importantes en la arena internacional: la diplomacia. Según Towns y Niklasson (2018, pp. 29-33), la mayoría de los países nombra pocas mujeres como sus embajadoras en misiones diplomáticas (los números oscilan entre ninguna embajadora en países como Arabia Saudita hasta el $41 \%$ en Filipinas y el $44 \%$ en Finlandia). 
jerarquías de género cementadas dentro de un sistema patriarcal más amplio, se percibe que lo que antes parecía una forma de protección del individuo es, en realidad, una estructura que define posiciones desiguales entre hombres y mujeres (Okin, 2008, pp. 320-324; Pateman, I988 [1993, Pp. 330-33I]).

La constitución de un mundo dividido entre dos espacios se sostiene en distintos argumentos sobre la naturaleza de los sexos, basándose en explicaciones biológicas y psicológicas que sitúan a las mujeres en un estado de permanente dependencia e inferioridad en relación a los hombres (Beauvoir, 1949[2019, pp. 2 I-24]; Hawkesworth, 2019, pp. 24-29; Pateman, 1988 [1993, Pp. 162-163]). La subordinación establecida en el ámbito doméstico, donde los jefes de familia tratan a las mujeres y sus hijos como subordinados, se transpone para el espacio que se encuentra afuera de lo doméstico, totalizando la experiencia de pertenencia a todas las formas de decisión y liderazgo en la sociedad (Pateman, 1988 [1993, pp. I37-143]). Como resultado de este proceso de jerarquización con base en el sexo ${ }^{3}$, se observa un aislamiento de las mujeres a una condición permanente de subordinación al hombre ( $y$, más ampliamente, al patriarcado) y de confinamiento en la esfera privada de lo doméstico. En esta esfera, las mujeres son responsables primariamente del cuidado de los miembros de la familia, la maternidad y todo aquello que se defina como la gestión de la casa. Aquí se establece una división sexual del trabajo, inherente al contrato sexual redactado y firmado por el patriarcado, la cual reserva a los hombres el espacio público de la política, la economía y la toma de decisiones.

Los movimientos feministas que emergen principalmente en el siglo XIX y se expanden en el siglo $X X$ denuncian la misión incompleta del estado y las revoluciones liberales, apuntando a la exclusión a la que están condenadas las mujeres. Aunque primeramente las demandas femeninas se hayan concentrado en la cuestión del voto y la participación política, rápidamente se extendieron para cuestiones de trabajo, economía e igualdad en todos los dominios donde imperan desigualdades entre hombres y mujeres. El cuestionamiento acerca de la división entre lo público y lo privado gana fuerza con la conocida consigna “lo personal es político", que pone en el centro de las discusiones las injusticias y las violencias que ocurren en la esfera privada, pero que se silencian bajo el contrato sexual del patriarcado (Heberle, 2016, pp. 596-604; Hooks, 2015 [2019, pp. 32-33]). El adviento del término género en las teorías feministas profundiza el debate acerca de cómo las jerarquías inciden sobre esta división (Scott, 20 I0, p. I0; Vigoya, 2016, Pp. 853856). Segato resume así:

“(...) la relación jerárquica que llamamos «género» como estructura binaria y desigual por la cual la posición masculina secuestra para sí la plataforma de enunciación de verdades de interés universal llamada «esfera pública» y se coloca en la posición de sujeto paradigmático de lo Humano pleno y englobante, en un gesto que expulsa a la posición femenina a la calidad de margen, resto, particularidad, cuestión de intimidad" (Segato, 2016, p. 83).

3 Son varios y distintos los mecanismos por los cuales se concreta la subordinación de las mujeres dentro del patriarcado. Las teorías feministas en las Ciencias Sociales y la Psicología ofrecen explicaciones sobre cómo se estructura la subordinación en diferentes dimensiones. Sin embargo, esta discusión extrapola el objetivo del artículo. Para más detalles acerca de este debate, ver: Miguel (2019), Okin (2008), Scott (20I0). 
Es precisamente en los roles de género donde se centran los primeros debates sobre la posición (y la ausencia) de las mujeres en el sistema internacional. La teórica J.Ann Tickner ( 1992 , 2006) señala que la arena internacional refleja estructuras de género presentes en el dominio doméstico y que son subyacentes al orden patriarcal más amplio y trasversal a las sociedades. Si en la política nacional el espacio público es ocupado por hombres, la arena internacional también lo es, caracterizándose como un dominio masculino y masculinizado. Bajo la mirada de Tickner y de otras teóricas feministas de las relaciones internacionales (Enloe, 2014, p. 31-32; Hooper, 200I, pp. 80-89; Runyan y Peterson, 20I4, p. I8, pp. I58-I63; Sylvester, 1996, pp. 264-265; Zalewski y Parpart, 2008, pp. 1-4), la agresividad del sistema internacional y el consecuente estado de belicismo inminente entre los países se basa en la esencia masculina que se transpone desde el plano doméstico hacia el internacional. Consecuentemente, los temas de seguridad, armamentos y guerra están bajo el control de los hombres, así como los cargos que se ocupan de estos temas y que son considerados más prestigiosos. Los pocos espacios que las mujeres ocupan en la escena internacional reflejan los estereotipos típicos del dominio doméstico, de los cuales lo más importante es el tema del cuidado. Las mujeres, por consiguiente, tendrían una naturaleza más adecuada para los temas de baja política (o sea, los temas de política internacional que estarían jerárquicamente en una posición inferior a los de seguridad y economía), eso cuando lleguen a acceder a cargos políticos internacionales (Monte, 20I3, p. 6I; Ventura y Kritsch, 2017, p. 35; Tickner, I992, p. 3;Tickner, 200 I, pp. 97-98, pp. I I0-I I I;Tickner, 2006, p. 278).

Sin adentrar en la discusión acerca del carácter esencialista de estas perspectivas teóricas, lo que ellas señalan como una característica del sistema internacional (o sea, la presencia mayoritaria de los hombres en los más altos y prestigiosos cargos políticos) se confirma ante los persistentes datos de baja presencia femenina como jefas de estado y gobierno, presidentas y vicepresidentas (y sus análogos) en organizaciones internacionales - como señalan Aggestam y Towns (2019, p. 9, p. 18) y Martins (2018, pp. 188-190)—, o jefas de delegación entre tantas otras posiciones en la esfera pública internacional. Asociada a esta baja presencia se verifica la continuidad de los estereotipos de género, confinando a las mujeres a determinados roles de baja política (Tickner, 2006, p. 278, pp. 280-282). Son pocos los ejemplos de mujeres que ocupan cargos en organizaciones militares y de defensa (Carreiras, 2009, pp. 170-173; Escobar, 2009, pp. 52-58; Mathias, 2009, pp. 8-9), o de economía y comercio; o asumiendo protagonismo en procesos de integración regional (Naurin y Naurin, 20 I8, pp. 220-231) y debates en foros y conferencias internacionales.

Esta ausencia de mujeres se puede remediar a través de los mecanismos propuestos por la perspectiva de la política de la presencia. Para la politóloga Phillips (1998), la subrepresentación de grupos excluidos de la esfera política se manifiesta como una forma de injusticia que consiste en conceder un status de virtud a determinados agentes dominantes a costa de aquellos a quienes les fue negada la presencia en los espacios de decisión.Ausentes, los excluidos no pueden llevar importantes contribuciones que emergen de sus experiencias vividas, lo que disminuye la capacidad del sistema político de responder a sus demandas. En este sentido, la presencia como instrumento político es fundamental para el empoderamiento de estos grupos, una vez que les permite vocalizar sus necesidades, intereses y, sobre todo, cosmovisión en el espacio público desde su perspectiva existencial (Castiglione y Pollak, 20I8, p. 5; Phillips, 20 I I, p. 344; Young, 2000, Pp. |4|-148). Además, estar presente cumple el más simbólico objetivo de la representación: demostrar que cualquier persona, independientemente de su género, puede llegar a ocupar los 
puestos políticos más altos, incluso en la arena internacional.

No sorprende, por lo tanto, que la misma cuestión de la presencia propicie un debate más amplio acerca de otras hendeduras sociales que excluyen a determinadas personas de los espacios públicos de decisión, incluso en la arena internacional. El feminismo negro acuña las formas interactivas de discriminación que emanan del enlace entre género, raza y clase de interseccionalidad (Cooper, 20 16, pp. 389-397; Crenshaw, I 99I, pp. I 242-I 245; Collins, 2000[2019, pp. 63-67]; Hooks, 2015 [2019, pp. 3I-34]), centrando su análisis en la condición especial de las mujeres negras en los países donde la colonización estableció jerarquías basadas también en concepciones raciales. Esta literatura informa las epistemologías feministas en Relaciones Internacionales a partir del reconocimiento de que la arena internacional sigue siendo un espacio dominado por hombres blancos de origen europeo, que llevan al espacio público internacional sus visiones raciales (Henderson, 20 I 5, pp. 38-40; Le Melle, 2009, pp. 78-82). Sumada a esta perspectiva, los feminismos del Sur Global (o feminismos subalternos) subrayan el papel de exclusión que los procesos de colonización han cementado a lo largo de la historia de América Latina, África y Asia (Ballestrin, 2017, p. 1038; Mendoza, 2016, Pp. 108-III; Segato, 2016, PP. 91-93). La permanente condición de subalternidad de los pueblos y, especialmente, de la mujer de los continentes antes colonizados les secuestra la voz y, sobre todo, su propia comprensión acerca de sus necesidades, demandas y cosmovisión (Lugones, 2008, pp. 92-99; Spivak, 1988[1998, pp. 27-29]). Eso resulta en que sus proyectos de nación y desarrollo sean silenciados por las perspectivas anglosajonas y eurocéntricas dominantes, al paso que promueve una inferiorización de las alternativas autóctonas de manejo de la política y economía. En esta escena de exclusión, las mujeres del Sur Global principalmente, aquellas que se encuentran en mayor desventaja debido a las interseccionalidades de raza y clase - padecen de violaciones específicas de sus derechos, que suelen ser ignorados si su presencia en el espacio público no está garantizado.

Es precisamente bajo esta óptica en la que se sitúa el cuestionamiento que anima esta investigación: si aceptamos que la presencia es importante como valor en sí mismo y como instrumento para que las mujeres, tradicionalmente excluidas de la esfera política internacional, puedan vocalizar sus demandas y cosmovisiones; y si la propia ONU, la organización internacional más importante, lo reconoce a través de sus políticas, resoluciones y discursos, se espera, por lo tanto, que a lo largo de las últimas décadas la participación femenina en sus órganos, especialmente en la calidad de jefas, se acerque de los ideales de una paridad de género. Este objetivo informa los debates más recientes acerca de la paridad de género, cristalizados en distintos tratados, convenciones y protocolos internacionales, demostrando la importancia de la participación política de las mujeres (Rubio-Marín, 2018, pp. 91-95), incluso en las estructuras del sistema internacional (Aggestam y Towns, 2018a, pp. I-2; Aggestam y Svensson, 2018, p. I49, pp. I55-I56, p. I59, p. I63; Balbino, 20II, pp. I67-191; Paffenholz, 2018, pp. I70-173). Eso es lo que investigaremos enseguida.

\section{Metodología}

En esta investigación, para responder a la pregunta inicial, partimos de un abordaje cuantitativo descriptivo basado en los datos de las Naciones Unidas y organismos del sistema económico global a lo largo de 1990 a 2018 . Estos datos están listados bajo dos variables: género y origen 
nacional. Con eso, proponemos dos hipótesis:

$\mathrm{HI}$ :Existe un déficit de la presencia femenina en los cargos de presidencia y/o vicepresidencia (y sus análogos) en los órganos y organismos especializados de las Naciones Unidas y en las instituciones del sistema económico global.

H2: Los organismos donde más mujeres han ascendido a los cargos de presidencia y/o vicepresidencia se ocupan de temas que refuerzan los roles y estereotipos de género.

En la tabla I, presentamos la lista de órganos y agencias de la ONU y sus respectivos temas y funciones. Añadimos, como forma de contemplar tres instituciones esenciales al sistema económico global, el Banco Mundial, el Fondo Monetario Internacional y la Organización Mundial del Comercio. Los datos fueron recolectados para cada una de las variables, de modo que se puedan reconocer dónde están las mujeres en términos de organismos y funciones desempeñadas.

Tabla I. Lista de órganos y organismos

\begin{tabular}{|l|l|}
\hline \multicolumn{1}{|c|}{ Órgano } & \multicolumn{1}{|c|}{ Funciones } \\
\hline Secretaría General & $\begin{array}{l}\text { Ofrece asistencia a otros órganos de las Naciones } \\
\text { Unidas y administra sus programas y políticas. }\end{array}$ \\
\hline Consejo Económico y Social (ECOSOC) & $\begin{array}{l}\text { Coordinación de los trabajos económicos y sociales, } \\
\text { de las agencias especializadas y de las demás } \\
\text { instituciones el Sistema de Naciones Unidas. }\end{array}$ \\
\hline Corte Internacional de Justicia (CIJ) & Brazo judicial de las Naciones Unidas. \\
\hline Asamblea General (AGNU) & $\begin{array}{l}\text { Espacio de deliberación entre todos los estados } \\
\text { miembros de Naciones Unidas. }\end{array}$ \\
\hline Consejo de Derechos Humanos (CDH) & $\begin{array}{l}\text { Aconseja a la Asamblea General acerca de temas de } \\
\text { derechos humanos y sus violaciones. }\end{array}$ \\
\hline \multicolumn{1}{|c|}{ Organismos Especializados y Programas } & Temas \\
\hline $\begin{array}{l}\text { FAO - Organización de las Naciones Unidas para la } \\
\text { Alimentación y la Agricultura }\end{array}$ & Agricultura y seguridad alimentaria \\
\hline $\begin{array}{l}\text { ICAO - Organización de la Aviación Civil } \\
\text { Internacional }\end{array}$ & Aviación \\
\hline IMO - Organización Marítima Internacional & Mares y navegación \\
\hline ITU - Unión Internacional de Telecomunicaciones & Telecomunicaciones \\
\hline Para la Educación, Ciencia y Cultura & Educación, Ciencia y Cultura \\
\hline $\begin{array}{l}\text { UNIDO - Organización de las Naciones Unidas } \\
\text { Desarrollo e industrias }\end{array}$ \\
\hline
\end{tabular}




\begin{tabular}{|c|c|}
\hline Órgano & Funciones \\
\hline OMT - Organización Mundial del Turismo & Turismo. \\
\hline PMA - Programa Mundial de Alimentos & Seguridad alimentaria. \\
\hline $\begin{array}{l}\text { OMPI - Organización Mundial de la Propiedad } \\
\text { Intelectual }\end{array}$ & Propiedad Intelectual \\
\hline OIT - Organización Mundial del Trabajo & Trabajo \\
\hline $\begin{array}{l}\text { UNCTAD - Conferencia de las Naciones Unidas } \\
\text { sobre Comercio y Desarrollo }\end{array}$ & Comercio y desarrollo \\
\hline $\begin{array}{l}\text { PNUD - Programa de las Naciones Unidas para el } \\
\text { Desarrollo }\end{array}$ & Desarrollo \\
\hline $\begin{array}{l}\text { ACNUR - Alto Comisionado de las Naciones } \\
\text { Unidas para los Refugiados }\end{array}$ & Refugiados \\
\hline $\begin{array}{l}\text { UNICEF - Fondo de las Naciones Unidas para la } \\
\text { Infancia }\end{array}$ & Infancia y cuidados \\
\hline IAEA - Organismo Internacional de Energía Atómica & Energía \\
\hline $\begin{array}{l}\text { PNUMA - Programa de Naciones Unidas para el } \\
\text { Medio Ambiente }\end{array}$ & Medio Ambiente \\
\hline $\begin{array}{l}\text { ACNUDH - Alto Comisionado de Naciones Unidas } \\
\text { para los Derechos Humanos }\end{array}$ & Derechos Humanos \\
\hline Organismos de la Economía Global & Temas \\
\hline BM - Banco Mundial & Finanzas, desarrollo y economía \\
\hline FMI - Fondo Monetario Internacional & Finanzas y economía \\
\hline OMC - Organización Mundial del Comercio & Comercio \\
\hline
\end{tabular}

Fuente: Elaboración propia.

\section{Resultados y discusión}

A lo largo de las tres décadas analizadas en esta investigación, la presencia femenina en los distintos cargos de Naciones Unidas y de las organizaciones del sistema económico global sigue bastante limitada. Entre 1990 y 2018 , treinta y cinco mujeres ocuparon las posiciones de mayor destaque en la organización, mientras ciento ochenta y seis hombres fueron presidentes, vicepresidentes o análogos. Estos números de pronto demuestran que las mujeres, en este ámbito de análisis, fueron el rostro del $18,82 \%$ de los órganos, organismos especializados y programas de las Naciones Unidas, además del FMI, Banco Mundial y de la OMC. 
Este déficit de representación se nota en toda la estructura de la ONU, desde la Secretaría General, que nunca ha sido conducida por una mujer, pasando por todos los distintos organismos. Aunque la presencia femenina se note en diferentes proporciones en esta investigación, el patrón de baja representatividad es claro, como demuestra la figura $I$.

Figura I. Número absoluto de mujeres en cada órgano u organismo, 1990-20 I 8

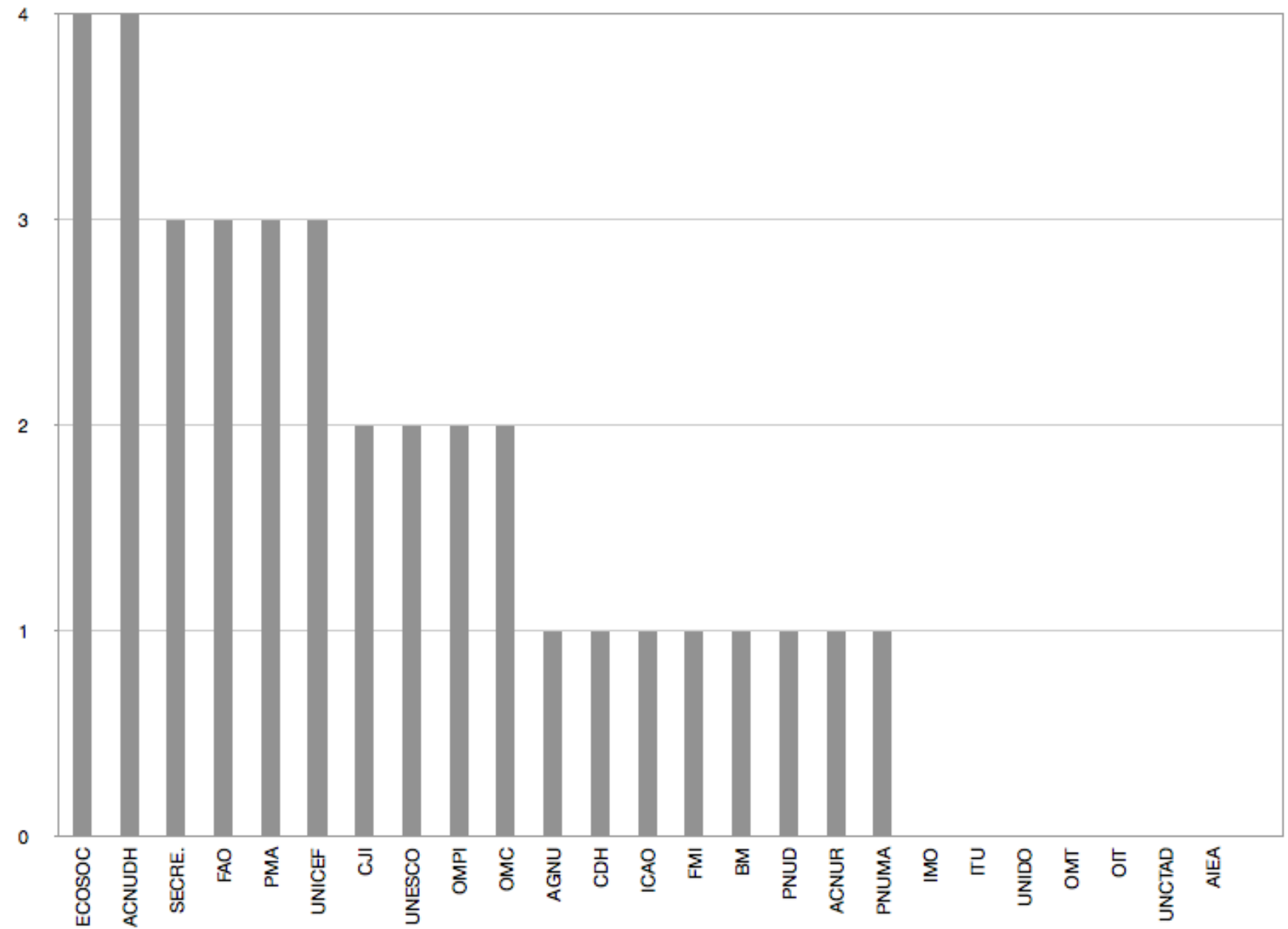

Fuente: Elaboración propia.

Esta desigualdad de representación debe tener en cuenta dos cuestiones. En primer lugar, los términos de presidencia y vicepresidencia de cada órgano, organismo y programa son distintos, lo que demanda que estos resultados se lean en perspectiva. Entre los casos analizados, el término más corto es el de ECOSOC, lo que resulta en un déficit más agudo: las mujeres han dirigido solo un $13,78 \%$ de los términos del órgano. Resultados semejantes se observan en los demás organismos analizados, como muestran los datos segregados y ponderados según sus respectivos términos en la tabla 2. 
Tabla 2. Presencia femenina en los altos cargos de los organismos de las Naciones Unidas y del sistema económico global, I990-20 I 8

\begin{tabular}{|c|c|c|c|}
\hline Organismo & Número absoluto de mujeres & Término [años] & Proporción respecto al año [\%] \\
\hline UNICEF & 3 & 3 & $60,00 \%$ \\
\hline PMA & 3 & 4 & $60,00 \%$ \\
\hline UNESCO & 2 & 4 & $50,00 \%$ \\
\hline ACNUDH & 4 & $4^{*}$ & $50,00 \%$ \\
\hline OMC & 2 & 4 & $40,00 \%$ \\
\hline SECRE & 3 & 5 & $33,33 \%$ \\
\hline PNUMA & I & 5 & $25,00 \%$ \\
\hline FMI & I & 4 & $25,00 \%$ \\
\hline ICAO & $\mathrm{I}$ & 5 & $25,00 \%$ \\
\hline PNUD & I & 5 & $20,00 \%$ \\
\hline OMPI & 2 & 6 & $20,00 \%$ \\
\hline ACNUR & $\mathrm{I}$ & 4 & $20,00 \%$ \\
\hline BM & I & 5 & $16,66 \%$ \\
\hline ECOSOC & 4 & I & $13,69 \%$ \\
\hline $\mathrm{ClJ}$ & 2 & 3 & $10,00 \%$ \\
\hline FAO & 3 & 3 & $7,69 \%$ \\
\hline $\mathrm{CDH}$ & I & I & $7,69 \%$ \\
\hline AGNU & I & I & $2,63 \%$ \\
\hline UNIDO & 0 & $6 * *$ & $0,00 \%$ \\
\hline IAEA & 0 & 4 & $0,00 \%$ \\
\hline IMO & 0 & 4 & $0,00 \%$ \\
\hline ITU & 0 & 4 & $0,00 \%$ \\
\hline OIT & 0 & 5 & $0,00 \%$ \\
\hline
\end{tabular}




\begin{tabular}{|c|c|c|c|}
\hline Organismo & Número absoluto de mujeres & Término [años] & Proporción respecto al año [\%] \\
\hline OMT & 0 & 4 & $0,00 \%$ \\
\hline UNCTAD & 0 & 4 & $0,00 \%$ \\
\hline
\end{tabular}

Fuente: Elaboración propia. *Promedio de 4 años; **Promedio de 6-7 años.

Al considerar la división entre alta y baja política, como proponen las teorías feministas tratadas anteriormente (Aggestam y Towns, 2018a, p. II;Tickner, 2006, p. 278), se verifica cómo los roles de género se refuerzan. Si consideramos que los temas de seguridad internacional y economía son típicamente más prestigiosos y considerados de alta política, y si les añadimos los altos puestos en la Asemblea General, la CIJ y la Secretaría General, notamos que solamente 33,33\% de los cargos de presidencia y vicepresidencia (y sus análogos) fueron ocupados por mujeres. Otra forma de leer estos mismos datos es que cuando ocupan puestos en las organizaciones internacionales y en el sistema de Naciones Unidas, a las mujeres se les reservan los temas de baja política, como medioambiente, derechos humanos, alimentos, cultura, educación e infancia.

Entre los distintos temas comportados por la baja política, se encuentran los que se asemejan a la concepción del cuidado que emana desde la esfera privada (Aggestam y Towns, 2018a, p. 6; Standfield, 2019, p. I52). Usualmente son temas que tratan de las funciones desempeñadas por las mujeres en el espacio doméstico, como el cuidado a la familia (principalmente, a los niños, ancianos, enfermos y discapacitados), la educación y la gestión de los alimentos. Serían esos asuntos de mujer los que les estarían legítimamente reservados en la arena internacional. Es precisamente cuando se utiliza la clave de género que se reconocen las relaciones de poder subyacentes a la estructura del sistema internacional, que condiciona la presencia femenina a determinadas áreas donde su conocimiento, oriundo de sus experiencias como mujer, es valorada y aceptada. Evidentemente, tal valoración está cementada en la propia jerarquía de género, que sostiene este orden en el cual las mujeres se responsabilizan primariamente por los temas menos prestigiosos y próximos de la condición de domesticidad (Standfield, 2019, pp. I53-I54).

Las constantes denuncias de tal realidad han contribuido para que el techo de cristal se rompa, aunque episódicamente. Los casos de Christine Lagarde (exdirectora gerente del FMI) y Kristalina Georgieva (expresidenta del BM y actualmente directora gerente del FMI) son ejemplos emblemáticos de mujeres que recientemente llegaron a ocupar altos puestos en instituciones de prestigio en el sistema internacional. Sin embargo, a lo largo del periodo investigado, la presencia de mujeres en estos cargos se caracteriza más como una concesión momentánea que un intento real de promoción de paridad de género en los altos cargos del sistema de Naciones Unidas y de los organismos de la economía global. En realidad, el rostro de estos cargos mantiene sexo, raza y dirección bien determinados: hombres blancos de los países desarrollados.

Esta denuncia es recurrente en la literatura feminista del Sur Global (Ballestrin, 2017, p. 1037; Grecco, 2020, pp. I35-I40; Mendoza, 20I8, pp. 108-III; Mohanty, 2003, pp. 58-63), y se orienta también a las mujeres que ocupan los pocos cargos que les son ofrecidos en las 
instituciones internacionales. Como ilustra la figura 2, la mayoría de las mujeres en los altos puestos proviene de la América Anglosajona y de Europa Occidental, correspondiendo al 55\% de toda la presencia femenina en el sistema de Naciones Unidas y los organismos económicos globales. Los países de África Subsahariana y América Latina responden por el 13\% de los cargos, pero lo que más llama la atención es la ausencia de mujeres de Oriente Medio y Norte de África. Eso se explica, posiblemente, por la situación de profunda exclusión de las mujeres de la esfera política en los países de mayoría islámica.

Figura 2. Representación regional de las mujeres en los altos cargos de las Naciones Unidas y organismos del sistema económico global, 1990-2018 [\%]

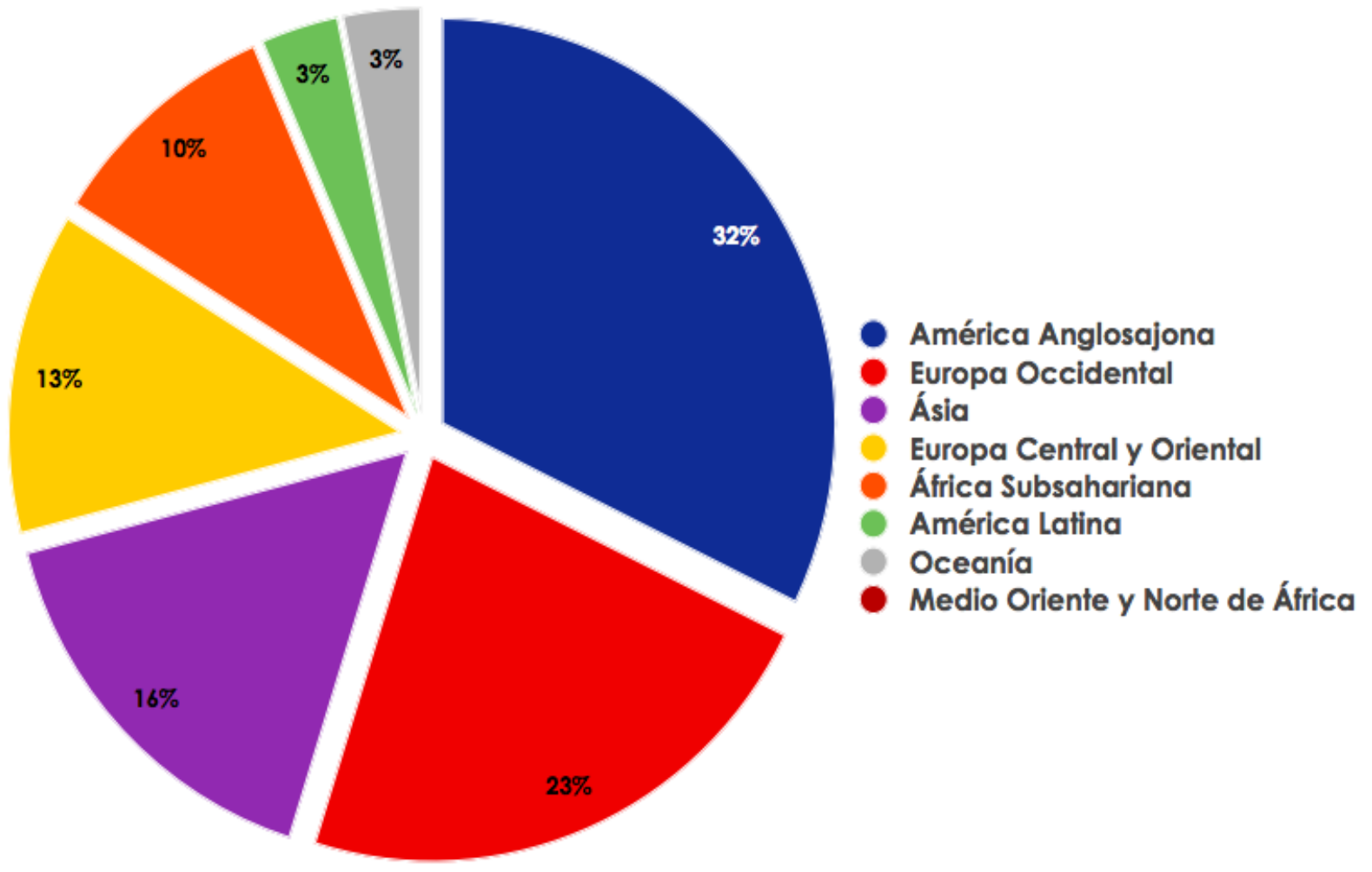

Fuente: Elaboración propia.

Estos hallazgos nos traen un diseño problemático de la situación de paridad de género en las Naciones Unidas y los organismos de la economía global, al paso que refuerzan resultados de investigaciones recientes acerca del tema (Aggestam y Towns, 2018b, p. 282; Aggestam y Towns, 2019, p. 18). A priori, la primera hipótesis ( $\mathrm{HI}$ ) propuesta anteriormente se confirma: el déficit de mujeres es un hecho que se pone en evidencia en el ámbito de esta investigación. El uso de simples datos cuantitativos permite verificar el estado de la presencia femenina, $y$ aunque el ideal 
de paridad de género pueda ser comprendido a través de fórmulas numéricas distintas ${ }^{4}$, lo que se nota es que las Naciones Unidas y los organismos económicos internacionales se alejan de cualquier perspectiva mínimamente paritaria. Además, la ausencia femenina es persistente en el tiempo analizado, de modo que no se trata de un "infeliz incidente": en realidad, el problema se revela como una exclusión sistemática, profundizada por los estereotipos acerca de las mujeres en la esfera política. Como señala Young, "todos los sistemas e instituciones de representación agrupan individuos según algún tipo de principios, y ninguno es inocente o neutro” (Young, 2000, p. I43).

Si rescatamos la perspectiva de la política de la presencia de Phillips (1998), la exclusión sistemática de las mujeres en los organismos internacionales sostiene una estructura dentro de la cual las demandas femeninas no son vocalizadas por sus representantes inmediatas.Al contrario, las demandas de las mujeres son conducidas en la arena internacional por representantes masculinos, que se encargan de concebir y manejar políticas y proyectos socioeconómicos en nombre de las mujeres. Eso contribuye al mantenimiento de la condición de subalternidad de las mujeres, al paso que consolida un permanente estado de ignorancia acerca de sus necesidades y perspectivas.

En cuanto a la $\mathrm{H} 2$, los resultados sugieren que la prevalencia de las (pocas) mujeres en los altos puestos de organismos refuerzan los estereotipos y roles de género (Aggestam y Towns, 2018b, pp. 283-288; Tickner, 2006, p. 278, pp. 280-282). La interpretación feminista de que las mujeres en la política internacional se encargarían de temas de baja política —porque bien poseen una esencia más cooperativa y pacifista, bien no estarían capacitadas para lidiar con la agresividad o supuesta complejidad de los temas de seguridad y economía - parece confirmarse ante los números en los organismos analizados. Además, la presencia recurrente en organismos que se ocupan de asuntos del cuidado (como por ejemplo UNICEF, UNESCO, PMA y ACNUDH) señala que el argumento de roles de género típicos del espacio doméstico se reproduce en la arena internacional. Como se ha mencionado anteriormente, estos datos dialogan con los números de ONU Mujeres (2020) acerca de los cargos ministeriales más frecuentemente ocupados por mujeres: persiste la predominancia de los asuntos sociales y del cuidado, desde la esfera de la política doméstica hacia la esfera de la política internacional.

Finalmente, el origen de las mujeres presidentas y vicepresidentas en los organismos investigados revela una realidad de intersección con el género: las que llegan a ocupar estos puestos provienen mayoritariamente de los países occidentales, principalmente de Europa y América del Norte. La ausencia más amplia de las perspectivas del Sur Global, y más específicamente, de las cosmovisiones y experiencias de las mujeres del mismo sur perjudican la proposición de políticas y acciones alineadas con las realidades locales. Subsiste dentro del feminismo incomodidad ante la mirada imperialista desde Europa y Estados Unidos en torno a las demandas de las mujeres del Sur -en verdad, este es el núcleo de la crítica de los feminismos subalternos/poscoloniales (Ballestrin, 2017, p. 1040; Lugones, 2008, pp. 92-94; Mendoza, 2016, pp. II4-II9) ${ }^{5}$. El desprecio

\footnotetext{
4 No hay consenso acerca de la fórmula exacta para que se reconozca un estado ideal de paridad de género. Para una discusión más profunda acerca de las implicaciones teóricas de la paridad de género, ver Fraser (2013). Para comprender la historia de los debates acerca de participación en la política y sus implicaciones empíricas y normativas, ver Rubio-Marín (2018).

5 Este incomodo ha sido manifestado desde las Conferencias Mundiales sobre la Mujer — como relatan Bonfiglioli (20I6) y Ghodsee (20I0) — y persiste ante los tratamientos basados en la lógica de que las mujeres del Sur Global necesitan ser salvadas de sus hombres, ignorando así los perniciosos efectos producidos por la colonización y legados como la estructura social, económica y política de estos países.
} 
por escuchar las voces subalternas refleja los prejuicios del propio sistema internacional ante los países del Sur Global y sus ciudadanos y ciudadanas.

Tal desprecio, sin embargo, impulsa la política neocolonial que enajena a estas mujeres de la elaboración de proyectos de desarrollo que se conducen en sus territorios. Al silenciar sus voces, se conciben ideales sociales, económicos y políticos que no dialogan con las necesidades de las poblaciones del Sur Global, y, más específicamente, de las mujeres que allí viven. Persiste la pregunta de Spivak sobre si puede hablar el sujeto subalterno: la respuesta, en el caso de los organismos internacionales, sigue siendo un categórico “¡No!”. Las consecuencias son conocidas principalmente en el caso de los programas de ajuste estructural del FMI (Sadasivam, 1997, p. 633; Subhash, 200I, pp. 6I-65).

En este escenario, en el cual la paridad de género suena más como un discurso de intenciones que como un camino que hacen juntos mujeres y hombres comprometidos con el cambio, vislumbrar salidas y acciones, se vuelve un reto per se. Sin embargo, los movimientos feministas han logrado importantes éxitos tanto en la arena doméstica como en la internacional, trayendo a la atención las diversas desigualdades que inciden directamente y con más fuerza sobre las mujeres. Como señala Miguel (2019, p. 53), "el feminismo tiene como objetivo explícito poner fin a una de las desigualdades más universales y duraderas de las existentes. La desigualdad sexual es también una profunda raíz material y psicológica de las que se nutren el resto de las desigualdades sociales". Quizás parte de la respuesta para corregir las aún persistentes ausencias femeninas en los altos cargos internacionales se encuentre en las demandas de mujeres que el propio feminismo, en sus distintas y plurales corrientes, se propone vocalizar. Generar un espacio de diálogo plural constante es la clave para que se impulsen políticas que abracen las realidades específicas de las diversas mujeres del mundo, tratando de las desigualdades que inciden sobre ellas teniendo en cuenta sus experiencias, necesidades e intereses.

Obviamente, eso presupone que los organismos internacionales tendrían que ofrecer algo más que discursos de empoderamiento de las mujeres, o que se los limite al contexto de negocios ${ }^{6}$. Reivindicar oportunidades para ocupar los altos cargos es esencial para que se concrete el ideal de igualdad de oportunidades, integración y no discriminación. Además, más mujeres no solo en esos puestos de presidencia y vicepresidencia, sino también en cargos de gestión de políticas son fundamentales para que se atienda a las distintas necesidades femeninas en diferentes contextos sociales, políticos y económicos. El empoderamiento, por lo tanto, demanda un compromiso más robusto de los organismos internacionales para con la paridad de género.

\section{Conclusión}

El activismo feminista se ha profundizado en el nuevo milenio, transformando los debates acerca de las desigualdades entre los sexos y las jerarquías de género subyacentes. Lo que antes, en los años setenta, parecía un esfuerzo hercúleo de establecimiento de conexiones entre feministas y

\footnotetext{
En 20II, ONU Mujeres estableció siete principios para el empoderamiento de las mujeres en el contexto de los negocios. Aunque se concentren primariamente en el contexto económico, los mismos principios se aplican al contexto político. Para más detalles, ver ONU Mujeres (20II).
} 
la vocalización de las demandas de las mujeres, hoy se ha convertido en un principio básico de la agenda internacional.

Investigamos la presencia femenina con datos de las tres últimas décadas, en los altos puestos de las Naciones Unidas y de las principales instituciones del sistema económico global. Verificamos que el techo de cristal sigue limitando la trayectoria profesional y política de las mujeres en la arena internacional, a pesar de los avances observados en términos de políticas promovidas por esas mismas organizaciones. Las pocas mujeres que acceden a las altas jerarquías internacionales aún enfrentan la soledad de un espacio dominado por hombres y por los prejuicios en contra de su capacidad de instrumentar, negociar y conducir políticas e instituciones. La sombra de la domesticidad parece seguirlas adónde vayan en la arena internacional.

En este artículo buscamos ofrecer los números que cementan un sentimiento ya conocido por feministas preocupadas con la representatividad de género en los organismos internacionales -incluso, por sus representantes, como el Secretario General António Guterres. El gran reto que se identifica a la luz de estos hallazgos es cómo desarrollar estrategias para empoderar políticamente a las mujeres, de modo que puedan acceder a los cargos de jefas en organismos internacionales, ministerios de relaciones exteriores y hasta diplomáticas. El simbolismo de la representatividad de ver a un rostro femenino en las instituciones, aunque pocas, es, seguramente, un paso fundamental en este proceso.

Evidentemente, las Naciones Unidas no son las únicas responsables de garantizar el acceso de mujeres a sus cargos. Esta misión depende de las decisiones de los estados miembros, que sugieren, respaldan y nombran los presidentes, vicepresidentes y sus análogos en estas organizaciones. Por lo tanto, el trabajo es más amplio, dado que requiere de una transformación mucho más profunda de las jerarquías de género al nivel nacional, específicamente en las carreras más próximas de la escena internacional. Bajo este contexto, no sorprende que una importante agenda de investigación feminista en Relaciones Internacionales se ocupe de las desigualdades de género dentro de las carreras diplomáticas, las cuales, en principio, ofrecerían los cuadros capacitados para conducir los organismos internacionales. Los hallazgos de tales investigaciones seguramente contribuyen a comprender la baja presencia femenina en las organizaciones internacionales, pero ellos extrapolan el objetivo inicial de este artículo.

El camino hacia la paridad de género es largo y apenas empieza. En lugar de desolación, los números muestran cuán grande es el reto que se abre ante nuestros ojos y nos invitan a seguir apuntando las desigualdades mientras buscamos remediarlas. Además, al comprender donde estamos en términos cuantitativos, somos llevados a investigar nuevas formas de evaluar los discursos manifestados por los líderes internacionales, los compromisos asumidos y los objetivos alcanzados.

\section{Referencias}

Adami, R. (2019). Women and the Universal Declaration of Human Rights. Routledge.

Aggestam, K. y Towns, A. E. (20 I 8a). Introduction:The Study of Gender, Diplomacy and Negotiation. En Aggestam, K. y Towns, A. E. (Eds.). Gendering Diplomacy and International Negotiation. Palgrave Macmillan.

Aggestam, K. y Towns, A. E. (20 I 8b). Conclusion:The Quest for Gender Justice in Diplomacy. En Aggestam, K.y Towns, 
A. E. (Eds.). Gendering Diplomacy and International Negotiation. Cham: Palgrave Macmillan.

Aggestam, K. y Towns,A. E. (2019). The gender turn in diplomacy: a new research agenda. International Journal of Feminist Politics, 2 I (I), 9-28. https://doi.org/I0.1080/I46I6742.2018.1483206

Aggestam, K. y Svensson, I. (2018). Where Are the Women in Peace Mediation? En Aggestam, K. y Towns, A. E. (Eds.). Gendering Diplomacy and International Negotiation. Palgrave Macmillan.

Balbino,V. R. (20I I). Diplomata. Substantivo comum de dois gêneros. Fundação Alexandre de Gusmão.

Ballestrin, L.M.deA.(20I7). Feminismos Subalternos. Estudos Feministas, 25 (3), I035-I 054.https://doi.org/I0. I 590/I806$9584.2017 \mathrm{v} 25 \mathrm{n} 3 \mathrm{p} 1035$

Beauvoir, S. de (2019). O segundo sexo (5 a edición). Nova Fronteira.

Biroli, F. (2018). Gênero e Desigualdades. Editorial Boitempo.

Bonfiglioli, Chiara (2016). The First UN World Conference on Women (1975) as a Cold War Encounter: Recovering Anti-Imperialist, Non-Aligned and Socialist Genealogies. Filozofija i Društvo, XXVII (3), 52I-54I. https://doi. org/I0.2298/FIDI60352 IB

Bunch, C. (20I2). Opening Doors for Feminism. Journal of Women's History, 24 (4), 2I3-22I. https://doi.org/l0.1353/ jowh.2012.0054

Carreiras, H. (2009). O Olhar dos Homens: Resistência e Cumplicidade nas Respostas Masculinas à Integração de Mulheres nas Forças Armadas. En Mathias, S. K. (ed.) Sob o Signo de Atena: Gênero na Diplomacia e nas Forças Armadas. Editora UNESP.

Castiglione, D. y Pollak, J. (2019). Introduction. En Castiglione, D. y Pollak, J. (eds.). Creating Political Presence. Chicago University Press.

Collins, P. H. (2019). Pensamento Feminista Negro. Boitempo.

Cooper, B. (2016). Interseccionality. En Disch, L. y Hawkesworth, M. (eds.). The Oxford Hanbook of Feminist Theory. Oxford University Press.

Crenshaw, K.W. (I99I). Mapping the Margins: Intersectionality, Identity Politics, and Violence against Women of Color. Stanford Law Review, 43 (6), I 24 I- 1299.

Enloe, C. (20I4). Bananas, Beaches and Bases: Making Feminist Sense of International Politics. University of California Press.

Escobar, L.T. (2009). Mulher, Forças Armadas e Missões de Paz na Região Andina. En Mathias, S. K. (ed.) Sob o Signo de Atena: Gênero na Diplomacia e nas Forças Armadas. Editora UNESP.

Fraser, N. (20I3). Fortunes of Feminism: From State-Managed Capitalism to Neoliberal Crisis. Verso.

Grecco, G. de L. (2020). Feminismo y género en los Estudios Internacionales. Relaciones Internacionales, 44, I 27 -I 45. https://doi.org/I0.I5366/relacionesinternacionales2020.44.007

Ghodsee, K. (2010). Revisiting the United Nations decade for women: Brief reflections on feminism, capitalism and Cold War politics in the early years of the international women's movement. Women's Studies International Forum, 33, 3-12. https://doi.org/10.1016/j.wsif.2009.1 I.008

Gouges, O. de (20I7). Declaración de los Derechos de la Mujer y de la Ciudadana. CAAW Ediciones.

Guterres, A. (2020). Mensaje del Secretario General: La disparidad de poder entre los géneros. Secretaría General de las Naciones Unidas.

Hawkesworth, M. (2019). Gender and Political Theory. Polity Press.

Heberle, R. (20I6). The Personal is Political. En Disch, L. y Hawkesworth, M. (Eds.). The Oxford Hanbook of Feminist Theory. Oxford University Press.

Henderson, E.A. (20I5). Hidden in plain sight: racism in international relations theory. En Anievas, A., Manchanda, N. y Shilliam, R. (Eds.). Race and Racism in International Relations: Confronting the global colour line. Routledge.

Hooks, B. (2019). Olhares Negros: Raça e Representação. Editora Elefante.

Hooper, C. (200I). Manly States: Masculinities, International Relations, and Gender Politics. Columbia University Press.

Lenine, E. y Oncampo, L. (2020). Recuperando a justiça de gênero e a África nas Conferências Mundiais da Mulher. Meridiano, 47, I- 19. https://doi.org/ 10.20889/M47e21009

Le Melle, T. J. (2009). Race in International Relations. International Studies Perspectives, 10 (I), 77-83. https://doi. org/I0.I I I I/j.I528-3585.2008.00359.x

Lugones, M. (2008). Colonialidad y Género. Tabula Rasa, 9, 73-101.

Mathias, S. K. (2009). Gênero, Defesa e Paz no Cone Sul. En Mathias, S. K. (Ed.). Sob o Signo de Atena: Gênero na Diplomacia e nas Forças Armadas. Editora UNESP.

Martins,A.P.M. (20I8). Reflexões sobre Igualdade de Gênero e os Organismos Internacionais. En Vitale, D.y Nagamine, R. (Eds.). Gênero, Direito e Relações Internacionais: Debates de um campo em construção. Salvador: EdUFBA.

Mendoza,B.(2018). Coloniality of Gender and Power:From Postcoloniality to Decoloniality. En Disch, L.y Hawkesworth, M. (Eds.). The Oxford Handbook of Feminist Theory. Oxford University Press.

Miguel,A. (2019). Neoliberalismo Sexual: El mito de la libre elección ( $12^{\mathrm{a}}$ edición). Ediciones Cátedra.

Mohanty, C.T. (2003). Feminism Without Borders: Decolonizing Theory, Practicing Solidarity. Duke University Press.

Monte, I. X. do (20/3). O debate e os debates: abordagens feministas das relações internacionais. Estudos Feministas, 2 I (I), 59-80. https://doi.org/I0.1590/S0I04-026X2013000100004

Naciones Unidas (2020). An open letter to the women of the world. Recuperado de: https://www.unspecial.org/20 I2/03/ an-open-letter-to-the-women-of-the-world/ (23.06.2020).

Naurin, D.y Naurin, E. (20I8). Descriptive Representation and Negotiation: Gender Balance in the Committees of the 
Council of the European Union. En Aggestam, K. y Towns, A. E. (Eds.). Gendering Diplomacy and International Negotiation (pp. 2I3-237). Palgrave Macmillan.

Oficina del Alto Comisionado de las Naciones Unidas para los Derechos Humanos - ACNUDH (2020). Vienna Declaration and Programme of Action. United Nations Human Rights. Office of the High Commissioner.

Okin, S. M. (2008). Gênero, o público e o privado. Estudos Feministas, I6 (2), 305-332. https://doi.org/I0.I590/S0 I04$026 \times 2008000200002$

ONU Mujeres (2016). El Progreso de las Mujeres en el Mundo 2015-2016:Transformar las Economías para Realizar los Derechos. Estudos Feministas, 24 (2), 589-6 I4. https://doi.org/I0.1590/I805-9584-20 I6v24n2p589

ONU Mujeres (0I.0I.2020). Mujeres en la política: 2020. Recuperado de: https://www.unwomen.org/-/media/ headquarters/attachments/sections/library/publications/2020/women-in-politics-map-2020-es. pdf?la=es\&vs=828 (I 2.04.2020).

ONU Mujeres (20II). Principios para el Empoderamiento de las Mujeres. Recuperado de: https://www.unwomen. org/-/media/headquarters/attachments/sections/library/publications/20I I/I0/women-s-empowermentprinciples_20II_es\%20pdf.pdf?la=en\&vs=I504

Paffenholz, T. (20I8). Women in Peace Negotiations. En Aggestam, K. y Towns, A. E. (Eds.). Gendering Diplomacy and International Negotiation. Palgrave Macmillan.

Pateman, C. (1993). O Contrato Sexual. Paz\&Terra.

Phillips, A. (1998). The Politics of Presence. Oxford University Press.

Phillips, A. (20II). O que há de errado com a democracia liberal? Revista Brasileira de Ciência Política, 6, 339-363.

Rubio-Marín, R. (2018).Women's Participation in the Public Domain Under Human Rights Law:Towards a Participatory Equality Paradigm Shift. En Rubio-Marín, R. y Kymlicka,W.(Eds.). Gender Parity \& Multicultural Feminism. Oxford University.

Runyan, A. S. y Peterson,V. S. (20I4). Global Gender Issues in the New Millennium (4ª Edición). Westview Press.

Sadasivam, B. (I997). The Impact of Structural Adjustment on Women: A Governance and Human Rights Agenda. Human Rights Quarterly, 19 (3), 630-665.

Scott, J. W. (2010). Gender: Still a Useful Category of Analysis? Diogenes, 57 (I), 7-I4. https://doi. org/I0.1I77/0392192110369316

Segato, R. L. (2017). La guerra contra las mujeres. Traficantes de Sueños.

Spivak, G. C. (1998). ¿Puede hablar el sujeto subalterno? Orbis Tertius, 3 (6), I-44.

Standfield, C. (2019). Feminist perspectives on diplomatic practice - a review. International Journal of Feminist Politics, 2 I (I), I52-I54. https://doi.org/I0.1080/146/6742.2018.1555005

Subhash, S. (200I). Structural Adjustment and Women in Third World. Journal of Social Science, 5 (I-2), 59-67. https:// doi.org/10.1080/09718923.2001.II892289

Sylvester, C. (1996).The Contributions of Feminist Theory to International Relations. En Smith, S., Booth, K.y Zalewski, M. (eds.) International Theory: Positivism and Beyond. Cambridge University Press.

Tickner,J.A. ( 1992). Gender in International Relations:Feminist Perspectives on Achieving Global Security. Columbia University Press.

Tickner, J.A. (200I). Gendering World Politics: Issues and Approaches in the Post-ColdWar World. Columbia University Press.

Tickner, J.A. (2006). Feminist Perspectives on International Relations. En Carlsnaes, W., Risse, T.y Simmons, B.A. (Eds.). Handbook of International Relations. SAGE.

Towns,A. E.; Niklasson, B. (20l8). Where are the Female Ambassadors? Gender and Status Hierarchies in Ambassador Postings. En Aggestam, K. y Towns, A. E. (Eds.). Gendering Diplomacy and International Negotiation. Palgrave Macmillan.

Ventura, R. W.; Kritsch, R. (2017). Relações Internacionais, Teorias Feministas e Produção de Conhecimento: Um Balanço das Contribuições Recentes. Monções, 6 (I I), 24-57. https://doi.org/ I 0.306 I 2/rmufgd.v6il I.6902

Vigoya, M.V. (2016). Sex/Gender. En Disch, L. y Hawkesworth, M. (Eds.). The Oxford Hanbook of Feminist Theory. Oxford University Press.

Wollstonecraft, M. (20I8). Vindicación de los derechos de la mujer. Ediciones Cátedra.

Young, I. M. (2000). Inclusion and Democracy. Oxford University Press.

Zalewski, M. y Parpart, J. L. (2008). Introduction: rethinking the man question. En Parpart, J. L. y Zalewski, M. (Eds.). Rethinking the Man Question: Sex, Gender and Violence in International Relations. Zed Books. 


\section{RELACIONES INTERNACIONALES}

Revista académica cuatrimestral de publicación electrónica Grupo de Estudios de Relaciones Internacionales (GERI)

Universidad Autónoma de Madrid, España

https://revistas.uam.es/relacionesinternacionales

ISSN 1699 - 3950

f facebook.com/RelacionesInternacionales

3. twitter.com/RRInternacional

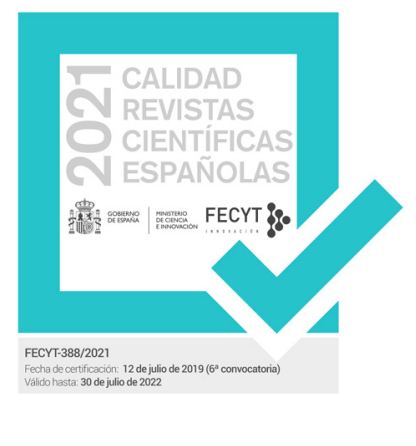

